\title{
The necessary and sufficient condition for the stability of a rigid body
}

\author{
W. S. Amer \\ Mathematics Department, Faculty of Science, Menofia University, Shebin El-Kom, Egypt \\ Email: drwaelamer@science.menofia.edu.eg
}

\section{Abstract}

In this paper, the stability of the unperturbed rigid body motion close to conditions, related with the center of mass, is investigated. The three first integrals for the equations of motion are obtained. These integrals are used to achieve a Lyapunov function and to obtain the necessary and sufficient condition satisfies the stability criteria.

\section{Keywords}

Rigid body, Stability, Lyapunov function, Euler-Poisson equations

\section{Introduction}

The stability of a rigid body has shed the attention of outstanding researchers during the last seven decades whether if the body contains fluid [1-4] or under the action of attracting center [5-13]. The stability of the rotational motion of a solid containing an ideal incompressible homogenous fluid filled entirely or partially was studied in [1] and [2]. This problem was investigated in [3] for the case of a uniform vortex motion, directed along a rough plane. The sufficient conditions for the stability of the studied motions are achieved. The permanent rotations and their stability for same problem were discussed in [4].

The stability of a rigid body, rotates about one fixed point, was discussed from different point of view such as in [5], the author studied the permanent rotation in the presence of Newtonian field, as in [6] when the motion is constrained similar to Euler's case and as in [7] for a certain special cases of the considered motion. Moreover, the first integral related with energy was used in [8] for the aim of obtaining the sufficient condition of the stability when the governing system of the body has not any perturbation. The stability of the steady motions of the rigid body problem when the body undergoes from potential field was investigated in [9] and generalized in [10] when the body is subjected to a central field whereas the stability of the spinning motion Lagrangian top was discussed in [11]. The orbital stability of a symmetric rigid body (pendulum oscillations and rotations) is carried out in [12] when the first two principal axes are equal twice of the third one. Recently, the permanent rotation of asymmetric rigid body was investigated in [13] when the body subjected to Newtonian field and a gyrostatic moment. The authors obtained the necessary and sufficient conditions, for some cases of the gyrostat configurations.

The main aim of this paper is to study the stability of the rotational motion of a rigid body for the unperturbed motion, relative to its variables, in the presence of supposed conditions associated with the center of mass. The first three integrals related with energy, area and the geometric one are obtained. By virtue of these integrals, the condition satisfies the stability criteria given by Lyapunov, is obtained which is considered necessary and sufficient condition.

\section{Statement of the problem}

Let $O$ be one of its fixed points of a rigid body coincides with the origin of a fixed frame $O X Y Z$ and a moving one $O x y z$. Let us consider that the body rotates with angular velocity $\underline{\omega} \equiv\left(\omega_{1}, \omega_{2}, \omega_{3}\right)$ about the principal axes of rotations $O x, O y$ and $O z$, and $\underline{\lambda} \equiv\left(\lambda_{1}, \lambda_{2}, \lambda_{3}\right)$ be the gyrostatic moment along the same axes in which the first component different from zero and the other two ones equal zero, see (Fig. 1). Bearing in mind that, the motion of the body is considered under the following conditions

$$
I_{1}>I_{2}>I_{3}, \quad x_{0} \neq 0, \quad y_{0}=z_{0}=0
$$

where $\left(I_{1}, I_{2}, I_{3}\right)$ represent the components of the principal inertia tensor $I$ of the body and directed along the principal axes, and $\left(x_{0}, y_{0}, z_{0}\right)$ refer to the components of the center of mass $C$. 


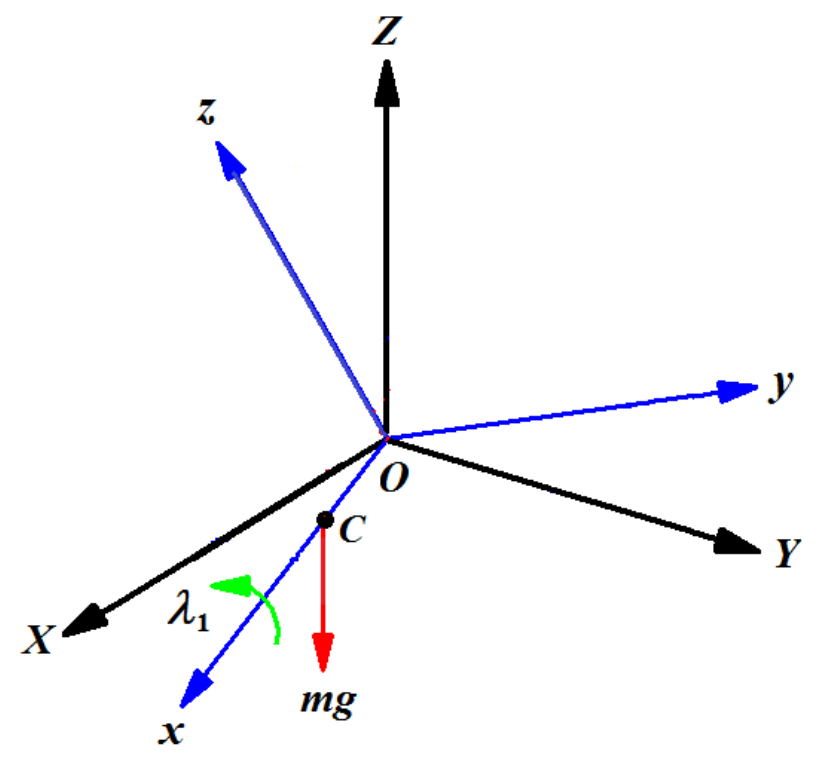

Fig. 1: Configuration of rigid body diagram

Therefore the Euler's dynamical equations for the motion of a heavy rigid body take the form

$$
\begin{aligned}
& I_{1} \dot{\omega}_{1}+\left(I_{3}-I_{2}\right) \omega_{2} \omega_{3}=0 \\
& I_{2} \dot{\omega}_{2}+\left(I_{1}-I_{3}\right) \omega_{3} \omega_{1}+\lambda_{1} \omega_{3}=m g x_{0} \gamma_{3}, \\
& I_{3} \dot{\omega}_{3}+\left(I_{2}-I_{1}\right) \omega_{1} \omega_{2}-\lambda_{1} \omega_{2}=-m g x_{0} \gamma_{2} .
\end{aligned}
$$

Here $\left(\gamma_{1}, \gamma_{2}, \gamma_{3}\right)$ denote to the components of the unit vector along the fixed axes $O Z, m$ represents the mass of the body, $g$ is acceleration due to gravity and dot dente to the derivative with respect to time $t$.

Considering $m g x_{0}=a$, consequently the previous equations can be rewritten in another equivalent form as

$$
\begin{aligned}
& I_{1} \dot{\omega}_{1}=\left(I_{2}-I_{3}\right) \omega_{2} \omega_{3}, \\
& I_{2} \dot{\omega}_{2}=\left(I_{3}-I_{1}\right) \omega_{3} \omega_{1}-\lambda_{1} \omega_{3}+a \gamma_{3}, \\
& I_{3} \dot{\omega}_{3}=\left(I_{1}-I_{2}\right) \omega_{1} \omega_{2}+\lambda_{1} \omega_{2}-a \gamma_{2} .
\end{aligned}
$$

The second system of the rigid body motion is the following Poisson's equations

$$
\begin{aligned}
& \dot{\gamma}_{1}=\omega_{3} \gamma_{2}-\omega_{2} \gamma_{3}, \\
& \dot{\gamma}_{2}=\omega_{1} \gamma_{3}-\omega_{3} \gamma_{1}, \\
& \dot{\gamma}_{3}=\omega_{2} \gamma_{1}-\omega_{1} \gamma_{2} .
\end{aligned}
$$

It is mention to state that, the governing equations of motion (2) and (3) have a special solution in the form 


$$
\begin{aligned}
& \omega_{1}=p=\text { const }, \quad \omega_{2}=\omega_{3}=0, \\
& \gamma_{1}=1, \quad \gamma_{2}=\gamma_{3}=0, \quad \lambda_{1}=0,
\end{aligned}
$$

in which characterizing the uniform rotation of the considered body about $x$-axis that coincides with the vertical.

\section{Stability procedure}

Now, we investigate the stability of the body for the unperturbed case relative to the variables $\omega_{1}, \omega_{2}, \omega_{3}, \gamma_{1}, \gamma_{2}, \gamma_{3}$

$$
\begin{array}{lcc}
\omega_{1}=p+\Gamma_{1}, & \omega_{2}=\Gamma_{2}, & \omega_{3}=\Gamma_{3}, \\
\gamma_{1}=1+\xi_{1}, & \gamma_{2}=\xi_{2}, & \gamma_{3}=\xi_{3} .
\end{array}
$$

Substituting (5) into (2) and (3) gives

$$
\begin{aligned}
& I_{1} \dot{\Gamma}_{1}=\left(I_{2}-I_{3}\right) \Gamma_{2} \Gamma_{3}, \\
& I_{2} \dot{\Gamma}_{2}=\left(I_{3}-I_{1}\right) p \Gamma_{3}+\left(I_{3}-I_{1}\right) \Gamma_{1} \Gamma_{3}-\lambda_{1} \Gamma_{3}+a \xi_{3}, \\
& I_{3} \dot{\Gamma}_{3}=\left(I_{1}-I_{2}\right) p \Gamma_{2}+\left(I_{1}-I_{2}\right) \Gamma_{1} \Gamma_{2}+\lambda_{1} \Gamma_{2}-a \xi_{2}, \\
& \dot{\xi}_{1}=\Gamma_{3} \xi_{2}-\Gamma_{2} \xi_{3}, \\
& \dot{\xi}_{2}=\left(p+\Gamma_{1}\right) \xi_{3}-\Gamma_{3}\left(1+\xi_{1}\right), \\
& \dot{\xi}_{3}=\Gamma_{2}\left(1+\xi_{1}\right)-\left(p+\Gamma_{1}\right) \xi_{2} .
\end{aligned}
$$

Referring to the above system (6), we can obtain the first integrals among its equations. To achieve this purpose, multiplying the first three equations of system (6) by $\Gamma_{1}, \Gamma_{2}, \Gamma_{3}$ and $\xi_{1}, \xi_{2}, \xi_{3}$ respectively, adding the results equations of each group and integrating the resulted two equations to obtain the first two integrals related with energy and area in the form

$$
\begin{aligned}
& V_{1}=I_{1} \Gamma_{1}^{2}+I_{2} \Gamma_{2}^{2}+I_{3} \Gamma_{3}^{2}+2 I p \Gamma_{1}+2 a \xi_{1}, \\
& V_{2}=I_{1} \Gamma_{1} \xi_{1}+I_{2} \Gamma_{2} \xi_{2}+I_{3} \Gamma_{3} \xi_{3}+I_{1} \Gamma_{1}+I_{1} p \xi_{1}+\lambda_{1} \xi_{1},
\end{aligned}
$$

where $V_{1}$ and $V_{2}$ represent the constants of integration.

The third first integral called geometric integral can be obtained after multiplying the last three equations of system (6) by $\xi_{1}, \xi_{2}, \xi_{3}$, adding the results equations and by integration, one obtains

$$
V_{3}=\xi_{1}^{2}+\xi_{2}^{2}+\xi_{3}^{2}+2 \xi_{1}
$$

in which $V_{3}$ is a constant. 
In the present work we will get the condition that satisfies the stability criteria as given by Lyapunov and Chetayev. Consequently, we construct Lyapunov function $L$ as the form of liner relation from integrals (7) as

$$
L=V_{1}-2 p V_{2}+\left(I_{1} p^{2}-a\right) V_{3}
$$

therefore, by virtue of (7) one gets

$$
L=L_{1}+L_{2}+L_{3}
$$

where

$$
\begin{aligned}
& L_{1}=I_{1} \Gamma_{1}^{2}-2 I_{1} p \Gamma_{1} \xi_{1}+\left(I_{1} p^{2}-a\right) \xi_{1}^{2}-2 p \lambda_{1} \xi_{1}, \\
& L_{2}=I_{2} \Gamma_{2}^{2}-2 I_{2} p \Gamma_{2} \xi_{2}+\left(I_{1} p^{2}-a\right) \xi_{2}^{2}, \\
& L_{3}=I_{3} \Gamma_{3}^{2}-2 I_{3} p \Gamma_{3} \xi_{3}+\left(I_{1} p^{2}-a\right) \xi_{3}^{2} .
\end{aligned}
$$

An inspection of (8c) shows that, the quadratic form $L_{1}$ is positive definite, it is necessary and sufficient that the following inequality is satisfied

$$
\left|\begin{array}{cc}
I_{1} & -I_{1} p \\
-I_{1} p & \left(I_{1} p^{2}-a\right)
\end{array}\right|=-I_{1} a>0
$$

consequently, one obtains

$$
a<0 \text {. }
$$

Similarly, the quadratic form $L_{2}$ is positive definite if

$$
\left|\begin{array}{cc}
I_{2} & -I_{2} p \\
-I_{2} p & \left(I_{1} p^{2}-a\right)
\end{array}\right|>0
$$

as a result of the previous determinant

$$
I_{2}\left(I_{1}-I_{2}\right) p^{2}-I_{2} a>0
$$

Moreover, the quadratic form $L_{3}$ is positive definite if 


$$
\left|\begin{array}{cc}
I_{3} & -I_{3} p \\
-I_{3} p & \left(I_{3} p^{2}-a\right)
\end{array}\right|>0
$$

which gives

$$
I_{3}\left(I_{1}-I_{3}\right) p^{2}-I_{3} a>0 .
$$

It is obvious that, conditions (10) and (11) are satisfied directly when condition (9) is satisfied for

$$
I_{1}>I_{2}>I_{3} .
$$

Therefore condition (9) is considered the necessary and sufficient condition for unconditional stability of the motion (4) with respect to the body variables $\omega_{1}, \omega_{2}, \omega_{3}, \gamma_{1}, \gamma_{2}, \gamma_{3}$.

\section{Conclusion}

The stability for the rotational motion of a rigid body about a fixed point when the center of mass lies on the third principal axes is carried out. The first integrals related with energy, area and the geometric one are achieved and used to construct the Lyapunov function. The necessary and sufficient condition satisfies the stability criteria is obtained.

\section{References}

[1] V. V. Rumiantsev, The stability of the rotational motions of a solid body with a liquid cavity, J. Appl. Math. Mech. 23, 6 , 1512-1524, 1959

[2] L. Li, On the stability of the rotational motion of a rigid body having a liquid filled cavity under finite initial disturbance, Appl. Math. Mech. 4, 5, 667-680, 1983.

[3] T. V. Rudenko, The stability of the steady motion of a gyrostat with a liquid in a cavity, J. Appl. Math. Mech. 66, 2, 171178,2002

[4] A. V. Karapentyan, V. A. Samaonov, T. S. Sumin, The stability and branching of the permanent rotations of a rigid body with a fluid filling, J. Appl. Math. Mech. 68, 893-897, 2004.

[5] G. K. Pozharitskii, On the stability of permanent rotations of a rigid body with a fixed point under the action of a Newtonian central force field, J. Appl. Math. Mech. 23, 4, 1134-1137, 1959.

[6] M. P. Guliaev, The stability of rotation of a rigid body with one point fixed in the Euler case, J. Appl. Math. Mech. 23, 2 , 579-582, 1959.

[7] lu. A. Arkhangelskii, On the stability of motion of a heavy solid about a fixed point in a certain special case, J. Appl. Math. Mech. 24, 2, 422-433, 1960.

[8] V. F. Liashenko, On the stability of a rigid body with a fixed point, J. Appl. Math. Mech. 30, 2, 499-502, 1967.

[9] V. D. Irtegov, On the problem of stability of steady motions of a rigid body in a potential force field, J. Appl. Math. Mech. 30, 5, 1113-1117, 1966.

[10] Ye. V. Abrarova, The stability of the steady motions of a rigid body in a central field, J. Appl. Math. Mech. 59, 6, 903 910, 1995.

[11] Yn. N. Kononov, Spin stability of a Lagrange top containing linear oscillators, J. Math. Sci. 103, 1, 2001.

[12] B. S. Bardin, A. A. Savin, The stability of the plane periodic motions of a symmetrical rigid body with a fixed point, J. Appl. Math. Mech. 77, 578-587, 2013.

[13] M. Iñarrea, V. Lanchares, A. I. Pascual, A. Elipe, Stability of the permanent rotations of an asymmetric gyrostat in a Newtonian field, Appl. Math. Comput. 293, 404-415, 2017. 